\title{
DIE PARTIKEL wie ALS VIELSEITIGE SATZEINLEITUNG
}

In dem Corpus von etwas mehr als 5500 Einzelsätzen aus populärwissenschaftlicher Prosa ${ }^{1}$, das als eine der Grundlagen für die Saarbrücker linguistischen Arbeiten dient, finden sich 482 Belege für die Partikel wie. Dieses reiche Material fordert dazu heraus, die verschiedenen Gebrauchsweisen der Partikel einer genaueren Betrachtung zu unterziehen.

Die Partikel wie kann Satzglieder und Gliedteile (unmittelbare und mittelbare Konstituenten) miteinander verknüpfen, sie kann ihnen auch in anderer Weise zugeordnet sein. Sie kann zur Anknüpfung von Nebensätzen dienen, und sie kann auch an der Spitze von Hauptsätzen stehen. Alle diese Gebrauchsweisen sind bekannt und werden in Grammatiken beschrieben. ${ }^{2}$ Sucht man aber angesich ts eines bestimmten Textbelegs in den Handbüchern Auskunft, so wird man oft enttäuscht. Manches ist unzulänglich, manches gar nicht beschrieben. In dieser Situation kann nur die Einzeluntersuchung weiterhelfen. Wenn hier die Partikel wie zum Gegenstand der Untersuchung gemacht wird, so möge das exemplarisch aufgefaßt werden. Es gibt einstweilen keine exakte Gesamtbeschreibung des syntaktischen Systems im heutigen Deutsch, und vermutlich trifft die vorliegende Untersuchung nicht einmal einen besonders wichtigen Punkt.

Immerhin besteht unseres Erachtens auf dem großen Gebiet der Syntax und Semantik der Nebensätze eine besonders empfindliche Forschungslücke. Da nun die Partikel wie mit ziemlich genau der Hälfte ihres im Corpus belegten Vorkommens satzeinleitend verwendet wird (21 Hauptsätze, 214 Nebensätze), kann gerade die Darstellung dieser Verwendung über sich hinaus und überhaupt auf die Problematik der Nebensätze hinweisen. Aus systematischen Gründen werden dabei auch die Hauptsätze mit wie in die Betrachtung einbezogen.

Die Satzbelege stammen, wie gesagt, aus populärwissenschaftlicher Prosa (Rowohlts Deutsche Enzyklopädie), geben also nur über eine bestimmte Textsorte Auskunft. Die Häufigkeitsangaben, die wir hinzufügen, sollen über die Verhältnisse in dieser Art schriftsprachlicher Prosa Aufschluß geben und einen Vergleich mit anderen Textsorten ermöglichen. 
Auf die Frage der Distribution, d.h. ob nur wenige oder eine Vielzahl der 50 Autoren, von denen die Belegsätze geschrieben wurden, an der betreffenden Gruppe von Belegen beteiligt sind, kann dagegen nur gelegentlich eingegangen werden.

Einige allgemeine Beobachtungen über den Gebrauch der Partikel wie seien vorausgeschickt. Sie kann adverbial alleinstehend oder auch als Modifikator (in vielen Grammatiken dann ebenfalls als "Adverb" bezeichnet) vor einem Adjektiv, Partizip oder Adverb verwendet werden. Diese Gebrauchsweise ist nahezu identisch mit der der sogenannten "Gradadverbien"; vgl. folgende Beispiele:

Er hat sich sehr gefreut.

Seine Freude war sehr groß.

Wie hat er sich gefreut. ${ }^{3}$

Wie groß war seine Freude.

oder in Nebensätzen:

..., wie er sich gefreut hat.

...., wie groß seine Freude war.

Gegenüber anderen Adverbien dieser Klasse besteht jedoch die Restriktion, daß wie, ob als Adverb oder als Modifikator, an der Spitze des Satzes stehen muß.

Spitzenstellung verlangt wie auch in jedem Falle, wenn es als satzeinleitende Konjunktion gebraucht wird:

Ich sah, daß er ankam.

Ich sah, wie er ankam.

Doch bedeutet das gegenüber den anderen Konjunktionen keinen Unterschied, da sie ja alle die Spitzenstellung verlangen.

Auch als glied- oder gliedteilverbindende Konjunktion unterscheidet wie sich nicht von anderen entsprechenden Konjunktionen:

Männer und Frauen wurden gerufen.

Männer wie Frauen wurden gerufen.

Es gibt gute und böse Beispiele.

Es gibt gute wie böse Beispiele.

Besonders häufig wird wie innerhalb des Satzes als Vergleichspartikel verwendet. Auf die vielseitigen Möglichkeiten in dieser Funktion kön- 
nen wir hier nicht eingehen. Der Gebrauch von wie in verschiedenen Gruppen von Vergleichsnebensätzen wird späterhin untersucht. ${ }^{4}$

\section{Wie an der Spitze von Hauptsätzen}

Nicht jedes wie an der Spitze eines Hauptsatzes ist von gleichem Interesse. In dem Satz

$3662^{5}$ Wie jedes Spiel muß man den Wettkampf als bis zu einem gewissen Grade zwecklos bezeichnen.

handelt es sich um einen Vergleich. Das wie ist hier nicht platzfest. Man kann die Konstituenten verschieben:

Den Wertkampf muß man wie jedes Spiel ...

Man muß den Wettkampf wie jedes Spiel ...

1. Hier geht es nur um Hauptsätze, in denen die Anfangsstellung des wie konstitutiv ist und nicht aufgegeben werden kann. Das ist bei Ausrufund Fragesätzen bestimmten Typs der Fall. Ausrufsätze sind in wissenschaftlicher und auch in populärwissenschaftlicher Prosa nur selten zu erwarten. Immerhin bietet das Corpus fünf Belege dafür. Dabei wird wie nur einmal alleinstehend als selbständiges Adverb verwendet:

6565 Wie alles sich zum Ganzen webt ...

Aber hier zitiert der moderne Autor Goethe. Der Beleg zeugt also nicht für die Gegenwartssprache, und ob adverbiales wie heute noch schriftsprachlich aktualisiert wird, kann man daraus nicht entnehmen. Denn die vier modernen Belege beweisen nur das modifizierende wie.

In einem Falle, in dem wie ein prädikatives Adjektiv modifiziert, ist jedenfalls wie in dem Goethe-Zitat die auffallende Endstellung des Verbum finitum zu beobachten:

1929 Wie zartbesaitet doch die Seele dieser Angelsachsen ist! ${ }^{6}$ Darf man aus dem Gegenbefund - Verbum finitum an zweiter Stelle bei nur drei Belegen Schlüsse ziehen, dann mit Zurückhaltung den Schluß, daß heute eine Tendenz zur "normalen" Hauptsatzstellung des Finitverbs besteht. ${ }^{7}$ 
Modifizierendes wie bei prädikativem Adjektiv liegt (wie in 1929) vor in:

8235 Wie schön sind die natürlichen Gebärden der schenkenden Huld ... !

Bindung an attributives Adjektiv:

8346 Und $^{8}$ wie viele Tierarten sind ... aus unserer Fauna verschwunden; ...

Modifizierung einer Adverbialgruppe liegt vor in:

1415 Wie ganz anders ist der... Mensch gebaut!

Nicht ganz unergiebig ist der Versuch, solche Ausrufsätze in Nebensätze zu transformieren, ohne ihren Sinngehalt zu ändern. Möglich wäre z.B.

Ist es nicht wunderbar (erstaunlich, bemerkenswert), wie schön die Gebärden ... sind?

Alle fünf Belege ließen sich in dieser Weise in einen Fragesatz einbringen. Aber jedesmal ist es keine echte, sondern eine rhetorische Frage. ${ }^{9}$ Eine den Sinn erhaltende Umwandlung ist nur im Aussagesatz möglich:

Es ist wunderbar, wie schön die Gebärden ... sind.

Man kann deshalb die Ausrufsätze in Verbindung zu jener Art von Nebensätzen bringen, die ich weiter unten ${ }^{10}$ versuchsweise als "modale Aussagesätze" bezeichnet habe.

2. Fragehauptsätze mit wie sind häufiger, nämlich sechzehnmal, belegt. 13 mal steht adverbiales wie allein an der Satzspitze, einmal ist es einem Adverb modifizierend zugeordnet ${ }^{11}$, und 2 Belege bedürfen in dieser Hinsicht der Diskussion. In jedem Fall ist es Bedingung, daß das wie an der Satzspitze steht.

Die größte Gruppe von 13 Belegen ist syntaktisch völlig durchsichtig; sie folgt Beispielen wie

50 Wie kam es dazu?

86 Wie sah sie aus?

$\mathrm{Da} B$ in ihnen aber ein semantisches Problem verborgen liegt, beweist die Ersatzprobe. In Satz 50 könnte man einsetzen: Auf welche Weise kam es dazu?, und der Charakter als Fragesatz bleibt dabei erhalten. Dieselbe Substitution ist in 9 weiteren Belegen dieser Gruppe möglich, nicht 
jedoch in Satz 86 und den beiden folgenden Belegen:

1234 Wie steht es aber mit dem zweiten Versuch?

1622 Und wie geht es ihm bei diesem Unternehmen?

Hier ist der Ersatz des wie durch eine andere fragende Konstituente überhaupt nicht möglich. Doch könnte die Umwandlung in Form einer "Antwort", also eines Aussagesatzes erfolgen: Sie sab gut (schön, wie eine Blume) aus. Auf jeden Fall ist hier eine Qualitätsaussage notwendig, also eine Qualitätsfrage gestellt, während in den anderen zehn Belegen eine Modalitätsfrage gestellt ist. ${ }^{12} 13$

Bei zwei Belegen für Modalitätsfragen liegen besondere syntaktische Verhältnisse vor:

4554 Und wie anders sollen wir die ... Besonderheiten ... deuten, die ... ?

1847 Wie sollte die Schwerkraft sonst zu verstehen sein? wo man auch die Kontaktstellung einsetzen könnte:

Wie sonst sollte...

Anderseits wäre in 4554 Distanzstellung möglich:

Wie sollen wir ... anders deuten, ...

Gewiß liegt in diesen Fällen zwischen wie und sonst, anders eine syntaktische Relation vor. Aber sie ist von anderer Art als z.B. Fügungen vom Typ wie weit, wie boch, wie lange, in denen wie das Adjektiv oder Adverb modifiziert und Kontaktstellung notwendig ist.

Sonst und anders hingegen sind in obigen Belegen Vertreter jener halbselbständigen, sekundären Satzglieder, die gern den Anschluß an eine übergeordnete Einheit aufsuchen. Auf jeden Fall ist in 1847 und 4554 wie der Kern der Gruppe, und nicht Modifikator.

Eindeutig als Modifikator ist wie in Fragehauptsätzen unseres Corpus nur ein einziges Mal belegt:

4480 Wie lange dauert es ..., bis ...

In solchen Fällen ist Kontaktstellung Bedingung. 


\section{Wie an der Spitze von Nebensätzen}

Bedeutend vielseitiger als bei den Hauptsätzen ist die Verwendung von wie zur Einleitung von Nebensätzen. Die Grammatiken pflegen abhängige Fragesätze, Relativsätze und Vergleichssätze zu unterscheiden, die entweder durch alleinstehendes wie oder durch modifizierendes wie eingeleitet werden können. Relativsä tze können nicht Hauptsätze sein ${ }^{14}$, und wenn Vergleiche im Hauptsatz vorkommen, so sind es sogenannte "Wortvergleiche"; ein Vergleichssatz im syntaktischen Sinne kann dagegen nur in der Form eines Nebensatzes auftreten. ${ }^{15}$

Wenden wir uns zunächst den Fragenebensätzen ("abhängige" oder "indirekte" Fragesätze) zu:

1908 Wie dies zuging, möge dem Tagebuch entnommen werden.

1496 Nachträglich ist schwer festzustellen, wie weit das stimmte.

Bei Umwandlung in einen Hauptsatz (Wie ging dies zu? Wie weit stimmte das?) tritt hier der Fragecharakter deutlich hervor. Damit unterscheiden sich die indirekten Fragesätze von einem andern Typ, der ihnen gleichwohl im allgemeinen zugezählt wird:

4298 Wie unrealistisch diese ... Auffassung ist, merkt man bei der Berufsberatung ...

1807 Sie bemerkte, wie die Unbeliebtheit des Kaisers wuchs.

Die Transformation in einen Fragehauptsatz (Wie wuchs die Unbeliebtbeit ... ?) würde, wenn sie überhaupt sinnvoll sein kann, doch jedenfalls den ursprünglich gemeinten Sinn verfälschen. Angemessen wäre dagegen die Umwandlung in einen Ausrufsatz:

Wie unrealistisch ist diese Auffassung.

Wie (sehr) wuchs die Unbeliebtheit des Kaisers. ${ }^{16}$

Man könnte in diesem Falle also "abhängige Ausrufsätze" annehmen, Da jedoch (mit leichter semantischer Modifizierung ${ }^{17}$ ) statt des wie hier auch ein $d a \beta$ eingesetzt werden kann:

Daß diese Auffassung unrealistisch ist, merkt man ...

Sie bemerkte, daß die Unbeliebtheit ... wuchs, 
halte ich es für richtiger, die Bezeichnung "modale Aussagenebensätze" (oder "abhängiger Modal-Aussagesatz") einzuführen.

Die Übergänge allerdings bleiben fließend. In Sätzen wie ... werden Störsignale gegeben, um festzustellen, wie stark die Reaktionen ... beeinträchtigt werden.

kann die Transformation sowohl einen Fragehauptsatz wie einen Ausrufsatz ergeben. In derartigen Zweifelsfällen muß es bei der Entscheidung auf Grund der Kompetenz des Beurteilenden bleiben. Dem Kontext gemäß stellen wir 3529 zu den Fragesätzen, da die Transformation in einen Ausrufsatz dem Zusammenhang nach nicht sinnvoll wäre.

Unter dieser Einschränkung, die dem subjektiven Urteil einen gewissen Spielraum läßt, stellen wir fest, daß im Saarbrücker Corpus 36 mit wie eingeleitete indirekte Fragesätze und 28 modale abhängige Aussagesätze vorkommen.

1. Wenden wir uns zunächst den abhängigen Fragesätzen zu. Die Transformationsprobe beweist, daß sie sämtlich sinnvoll (d.h. dem Kontext Rechnung tragend) in Fragehauptsätze umgewandelt werden können. In 28 Fällen leitet wie allein den Fragenebensatz ein ${ }^{18}$, achtmal steht es modifizierend vor einem Adjektiv oder Adverb.

Betrachtet man die Plazierung des abhängigen Fragesatzes im Gesamtsatz, so ergibt sich, daß 6 Belege, alle durch wie allein eingeleitet ${ }^{19}$, das Vorfeld füllen, wie oben durch Satz 1908 dargestellt. In 17 Belegen, darunter vier mit modifizierendem wie (vgl. oben 1496), steht der abhängige Fragesatz am Ende des Feldes, also dem Hauptsatz nachgestellt. Relativ häufig, nämlich in 13 Belegen, davon vier mit modifizierendem wie, ist der indirekte Fragesatz im zweiten Unterordnungsgrade von einem Nebensatz oder Infinitiv abhängig, steht aber auch dann am Ende des Satzgefüges. ${ }^{20}$ Als Beispiel sei sogar eine Unterordnung dritten Grades angeführt:

8523 Und man kann diese Rituale vollziehen, weil die Götter sie ... offenbart haben, indem sie ... den Menschen zeigten, wie man diese Nährpflanze anbaut ...

Denkbar wäre in manchen Fällen Einschub in das Satzfeld. Man könnte den oben zitierten Satz 1496 z.B, in die Folge bringen:

Nachträglich ist, wie weit das stimmte, schwer festzustellen. 
Eine solche Fügung würde allerdings als preziös und hochstilisiert empfunden werden. Es ist daher kein Zufall, daß in der nüchternen populärwissenschaftlichen Prosa eine derartige Einschub-Stellung niemals, sondern ausschließlich Vorfeld- oder Satzendstellung vorkommt, wobei letztere deutlich bevorzugt wird ( 30 gegen 6 Belege).

Syntaktisch relevant ist weiterhin die Frage, ob ein Nebensatz als Satzglied des übergeordneten Satzes interpretiert werden kann. So sind beispielsweise die oben in 1908 und 1496 zitierten wie-Sätze im Verhältnis zum Hauptsatz als Subjekte anzusehen, in 1807 und 4298 stellen sie dagegen Objekte dar, ebenso in 3529. Insgesamt sind 10 Subjektsätze (davon drei vorfeldfüllend) und 15 Objektsätze (drei im Vorfeld) vorhanden. Ferner finden sich acht Adverbialsätze, alle am Ende des Satzfeldes. Hierher rechne ich Belege wie

3703 Die Geschichte wird darüber befinden, wie oft sein Urteil richtig ... war.

4260 Es geht darum, wie Menschen sich selbst ... sehen.

In aller Regel dient in solchen Fällen ein Adverb im übergeordneten Satz als deiktisch-steuernder Bezug, der dem wie-Satz seine Relation zum Hauptsatz zuweist. ${ }^{21}$ Dabei ist die Substitution eines nominalen, mit Präposition ausgestatteten Gliedes möglich (über die Richtigkeit seines Urteils - um die eigene Sicht [Eigensicht, Selbstsicht] des Menschen).

Insgesamt 33 Belege zeigen also den indirekten Fragesatz als "Gliedsatz". Drei Belege lassen sich aber nicht so einordnen. Sie folgen dem Typ

4439 Die Untersuchung ... schließt ... die Frage ein, wie Ich und Welt in Erscheinung treten. ${ }^{22}$

Hier ist der wie-Satz eindeutig Attribut zu die Frage, also nur zugeordneter Teil eines Satzgliedes, und mithin "Gliedteilsatz", oder - wie wir der Kürze halber fortan schreiben wollen - "Teilsatz". ${ }^{23}$

2. Beim modalen Aussagenebensatz liegen die Verhältnisse insofern anders, als 19 Belege durch modifizierendes wie eingeleitet werden, und nur 9 durch alleinstehendes wie. Fünfmal füllt der wie-Satz das Vorfeld (2 Subjekt-, 3 Objektsätze), $21 \mathrm{mal}$ steht er am Satzende, davon $8 \mathrm{mal}$ nach Infinitiv (4) oder Nebensatz (4). ${ }^{24}$ Davon entfallen 6 Belege auf die Subjekt- und 15 auf die Objektsätze. Adverbialsätze kommen un- 
ter den Modalaussagesätzen überhaupt nicht vor.

Von den beiden hierher gerechneten Attribut- (also Teil-)sätzen weist der eine wieder Distanzstellung am Satzende auf:

8799 ... eine Kunst ..., deren Rangstufe sich nach dem Maß bestimmt, wie alle Bauelemente ... zueinander in das richtige Verhältnis gesetzt sind.

In dem zweiten Falle ist Einschub in das Vorfeld zu beachten, wobei sich die Kontaktstellung von selbst ergibt:

$6133 \ldots$ und die Erkenntnis, wie verschieden diese (Anschauungen) sein können, würde schon viel ... beitragen.

Als Kriterium für die Zuweisung eines wie-Satzes zu den Modalaussagen wurde oben bereits die Möglichkeit der Transformation in einen Ausrufsatz angegeben, die z.B. vorliegt in:

6590 Wie verschieden die Welten geworden waren, zeigte die Propaganda ... - (Wie verschieden waren die Welten geworden!)

Diese Transformationsmöglichkeit besteht bei allen 19 Belegen für modifizierendes wie. Von den neun Belegen mit alleinstehendem wie bietet nur ein Satz diese Möglichkeit:

8372 Wie die parlamentarische Demokratie in Norwegen wirtschaftete, mißfiel ihm.

Besser als durch den Ausrufsatz (Wie wirtschaftete ...!) würde hier allerdings der Sinn der Aussage durch die Erweiterung die Art, wie ... wiedergegeben. Transformation in einen $d a \beta$-Satz ist in 8372 jedenfalls nicht möglich, während die andern 8 Belege sie erlauben. Dem oben (S. 164) gegebenen Beispiel (wie / daß die Unbeliebtbeit wuchs) fügen wir noch hinzu:

6563 So sehen wir, wie die allseitige Verflechtung ... über die Erde hinausgreift ...

Mit dem Ersatz des wie durch ein daß ist allerdings stets eine semantische Nuancierung verbunden: wie betont den Verlauf des Geschehens und wirkt durativ, daß hingegen hebt das Faktum selbst hervor und wirkt punktuell.

Auch statt des modifizierenden wie-Satzes lassen sich daß-Sätze bilden, wobei aber ebenfalls eine Sinnverschiebung eintritt. In 6590 z.B. stellt 
die Fügung

wie verschieden die Welten geworden waren

den Grad der Verschiedenheit heraus, während

daß die Welten (sehr) verschieden geworden waren

das Faktum konstatiert. ${ }^{25}$ Eine semantisch gleichwertige Ersetzbarkeit durch daß liegt also bei beiden Gruppen der modalen Aussagesätze nicht vor.

Exkursweise seien an dieser Stelle die vier im Corpus vorhandenen Belege für die Fügung die Art, wie behandelt:

3649 Über die Art, wie das Chinesische die Spielfunktion ausdrückt, hat mir Professor D. ... Auskünfte gegeben.

Den wie-Satz möchte ich hier als indirekten Fragesatz klassifizieren. Möglich wäre: Darüber, wie ... ausdrückt, bat ... oder auch Wie ... ausdrückt, darüber bat ... Man könnte deshalb geneigt sein, die Fügung über die Art als ein deiktisch steuerndes Element nach Analogie der oben S. 166 erwähnten darüber, darum anzusehen. Dasselbe wäre möglich in

8231 ... daß man über die Art, wie Athene ... mit Hektor umgeht, nichts Besseres zu sagen weiß, als ... ,

wo übrigens eher ein modaler Aussagesatz (Transformation in einen Ausrufsatz ist möglich) vorliegt. In einem weiteren Beleg für den modalen Aussagesatz:

8285 ... einige sind schwer enttäuscht worden durch die Politik oder durch die Art, wie sie geführt wurde ...

wirkt der Ersatz durch dadurch, wie einigermaßen ungeschickt. Vollends unmöglich ist er in

4309 Die Art, wie solche ... Vorstellungen zustande kommen, ist ... kaum erforscht ${ }^{26}$,

weil hier nämlich die Art im Nominativ und nicht in einem Präpositionalkasus steht. Aber deutlicher noch als in den Präpositionalfügungen ist die Art hier überhaupt entbehrlich:

Wie solche Vorstellungen zustande kommen, ist kaum erforscht wäre möglich, womit wir zu einem eindeutigen indirekten Fragesatz 
und, auf den Hauptsatz bezogen, zu einem Subjektsatz gelangen.

Hier zeigt sich also eine Erscheinung, wie sie auch bei den $d a \beta$-Sätzen zu beobachten ist. Daß er kommt, freut mich ist ebenso möglich wie Die Tatsacbe, daß er kommt, freut mich, wo wir es einmal mit einem Subjekt-, also Gliedsatz, und das andere Mal mit einem Attributsatz, also einem Teilsatz, zu tun haben. Diese Unterscheidung ist auch bei Fügungen mit oder ohne die Art (wie) aufrechtzuerhalten. Ist die Art vorgeschaltet, so haben wir es - je nach dem semantischen Gehalt zwar auch mit indirekten Fragesätzen oder mit modalen Aussagesätzen zu tun, aber nicht mit Gliedsätzen, sondern mit (attributiven) Teilsätzen. Wir können also je zwei der eben behandelten Belege den beiden Nebensatzarten zurechnen und kommen damit für unser Corpus auf die Anzahl von 38 indirekten Fragesätzen und 30 modalen Aussagenebensätzen.

Eine für den heutigen Sprachgebrauch nicht unwichtige Beobachtung sei hier noch angefügt. Bei vergleichenden wie-Sätzen ist häufig ein sogenanntes "Korrelat" anzutreffen. ${ }^{27}$ Möglich wäre es in jedem Fall auch bei indirekten Frage- und Modalaussagesätzen, wenn diese im Vorfeld des Hauptsatzes stehen, also bei insgesamt 12 Belegen. Man könnte z.B. schreiben: Wie dies zuging, das möge man dem Tagebuch entnebmen. Ein solches Korrelat ist aber nirgends aktualisiert, mit folgender Ausnahme in einem stark rhetorischen Beleg:

8360 Wie im Grunde diese 'gemeinschaftsdienliche Zweckmäßigkeit' ... und wie diese ... Harmonie und Ordnung zu verstehen sind, dies zu untersuchen, ist nicht ... unsere Sache.

Als korrelierendes Pronomen kommt in der Regel nur das (der, die) in Frage. Die stärkere Deixis, die sich in dies ausdrückt, läßt erkennen, daß der Autor nach dem langen Anlauf sozusagen Atem schöpft und mit dies neu ansetzt.

3. Einschränkungssätze können durch wie aucb (immer) oder diskontinuierlich durch wie ... auch eingeleitet werden. Sie können dem Hauptsatz (oder einem Satzgefüge) folgen. Ob die vier Belege des Saarbrücker Corpus, die sämtlich Anfangsstellung zeigen, einen bevorzugten heutigen Sprachgebrauch wiedergeben, kann wegen ihrer geringen Anzahl nicht mit Sicherheit gesagt werden. Auch hier kann wie allein- 
stehend oder modifizierend bei einem Adjektiv (o.ä.) vorkommen. Die Belege lauten:

8273 Wie auch immer die Erklärung lauten mag, eine empirische Untersuchung muß stets vorgenommen werden.

3806 Wie dem auch gewesen sein mag, wo immer Julian von ihm spricht, geschieht es ohne Zuneigung.

6159 Wie es auch mit der Ehe ausgehen mag - die Frauen bleiben ihr Leben lang im Hause ihrer Sippe ...

8299 Wie aufschlußreich und wichrig auch der Nachweis ... sein mag ... - gerade das Wesentliche ... wird ... nicht beleuchtet.

Die vier Belege zeıgen übereinstimmend ein sehr charakteristisches Merkmal: Sie stehen isoliert außerhalb des Vorfeldes. Das ist besonders auffällig in 3806, wo das Vorfeld durch den Nebensatz wo immer ... spricht eingenommen wird. Ferner ist durch keinerlei Ersatzprobe der einschränkende wie-Satz in ein Glied, geschweige denn einen Gliedteil (Attribut) des übergeordneten Satzes einzuordnen, ja, es gibt gar keinen syntaktisch übergeordneten Satz. ${ }^{28}$

An anderer Stelle habe ich ausgeführt, weshalb es angebracht ist, den Terminus "Nebensatz" beizubehalten und als Oberbegriff für Gliedund Teilsätze sowie für "weiterführende" Nebensätze zu verwenden. ${ }^{29}$ Hier kommt nun eine vierte Subklasse der Nebensätze zum Vorschein: Wir haben es weder mit Glied- oder Teilsätzen noch auch mit weiterführenden Nebensätzen ${ }^{30}$ zu tun. Wegen ihrer syntaktischen Ungebundenheit möchte ich sie als "isolierte Nebensätze" bezeichnen. ${ }^{31}$

Wenn wir nunmehr zu der Funktion des wie als Vergleichspartikel übergehen, so stoßen wir auf eine große Anzahl von Satzbelegen, die in den meisten Grammatiken unter der Bezeichnung "Vergleichssätze" behandelt werden. Näheres Zusehen zwingt aber auch hier zur Unterscheidung einer Anzahl von Subklassen, und wir werden zwischen Vergleichssätzen, vergleichenden Relativsätzen und Hinweissätzen zu differenzieren haben.

4. Zwei Belege mögen zunächst für den Vergleichssatz im engeren Sinne stehen: 
6300 Wie ein Feinschmecker sich aus dem Kuchen nur die Rosinen heraussucht, so hat die Zecke aus den Dingen ihrer Umgebung nur die Buttersäure herausgelöst.

3934 Alles das ist freilich ... nicht ganz so ungewöhnlich, wie es zunächst scheinen mag.

In 6300 haben wir es mit einem sogenannten "Satzvergleich" zu tun. Während der "Wortvergleich" zwei Einzelvorstellungen zueinander in vergleichende Beziehung setzt ${ }^{32}$, wobei es nicht zur Ausbildung eines vergleichenden Nebensatzes kommt, wird im Vergleichssatz ein satzmäßig formulierter Zusammenhang zum Vergleich mit einem ebenso formulierten übergeordneten Satz herangezogen. Wie-Sätze dieser Art sind immer Adverbialsätze, also Gliedsätze, wenn auch die Transformation in ein nominales Satzglied wegen des komplexen Vorstellungsgehalts oft zu ungebräuchlichen, überladenen Fügungen führt. ${ }^{33}$ Es kann auch vorkommen, daß eine Nominalisierung aus Mangel an Wortmaterial überhaupt nicht möglich ist, so etwa in

1110 Das ist auch so, wie es sein sollte.

Aber das ändert nichts an der adverbialen Funktion des wie-Satzes. Ihrem Gehalt nach könnte man die Gruppe, zu der 1110 und 6300 gehören, als "Satzvergleichssätze" bezeichnen, ihrer syntaktischen Funktion nach als "vergleichende Adverbialsätze".

Ein anderer Typ liegt in 3934 vor, wo der - auch hier satzmäßig formulierte - Vergleich sich auf die Adjektivgruppe so ungewöbnlich bezieht. Erst durch die Bindung an das Adjektiv wird in derartigen Fügungen der wie-Satz in den Gesamtsatz integriert. Man hat ihn also als Teil der Adjektivgruppe zu betrachten, als Teilsatz also, und zwar als Attributsatz. ${ }^{34}$ Auch die Verschiebeprobe spricht für diese Auffassung. Denn die gesamte Adjektivgruppe ließe sich im Vorfeld des Hauptsatzes unterbringen, wo ja nach landläufiger Auffassung ${ }^{35}$ nur ein einziges Satzglied Platz hat:

So ungewöhnlich, wie es scheinen mag, ist das alles nicht.

Der adverbiale Vergleichssatz ist, wenn wir diesen Schluß aus unserem Corpus-Material ziehen dürfen, beweglicher als der attributive. Neunmal ist er in Vorfeldstellung (vgl, oben 6300) belegt, dreizehn Belege zeigen ihn am Ende des Satzfeldes (wie oben 1110). Einmal ist er auch in das Satzfeld eingeschoben: 
3717 Den Vorgang hat ... Hopkins so, wie er ihn ... erzählt bekam, aufgezeichnet.

Auch in einem Nebensatz ist er einmal eingeschoben:

8455

... daß er, wie wir heute in ähnlichen Fällen tun, sich damit begnügte, dem Ding einen Namen zu geben ...

Der Satz 8455 enthält nicht das Korrelat so zum wie-Satz, und es wäre auch in den oben zitierten Belegen 6300, 1110 und 3717, ja überhaupt in sämtlichen 24 Adverbialsätzen dieser Gruppe entbehrlich. Trotzdem ist in 20 Belegen dieses redundante so (bzw. ebenso, nur ein Beleg) vorhanden. Es übernimmt die deiktische Funktion, dem wie-Satz einen Platz als Adverb im übergeordneten Satz anzuweisen. Die häufige Verwendung im heutigen Schriftdeutsch - oder jedenfalls in dieser Textsorte - ist wohl zunächst einem Streben nach Deutlichkeit zuzuschreiben. Allerdings scheint diese Tendenz bereits zu formelhafter Starrheit zu führen, so daß die Gruppe so wie als satzeinleitende Einheit empfunden wird, wie die Zeichensetzung lehrt. Die Interpunktion so, wie wie oben in Satz 1110 - ist nämlich nur dreimal belegt. Dagegen kommt so wie:

6114 Die Größe der Ellipsen könnte ... variieren, so wie die Bahnen der Planeten verschieden sind.

sechsmal vor. ${ }^{36}$ Solcher Pleonasmus wird besonders deutlich, wenn der Vergleichssatz im Vorfeld steht:

4705 So wie hier thermische Größen geregelt werden, lassen sich auch mechanische ... regeln.

Im attributiven Vergleichssatz ist das so (bzw. ebenso; 4 Belege) konstitutiv unentbehrlich, da hier immer ein graduierendes Moment beteiligt ist und durch so bezeichnet wird. Damit der Vergleich in der Aussage verständlich wird, muß das durch so modifizierte Adjektiv (es kann prädikativ, adverbial oder attributiv gebraucht sein) in der Regel vorangehen. Daher mag es sich erklären, daß in den insgesamt acht Belegen, wie z.B. oben in 3934, der attributive Vergleichssatz stets am Ende des Satzfeldes steht. Unbedingt notwendig ist das nicht. So könnte z.B. in dem Beleg

$6530 \quad$... daß die Abstraktion dieser Erscheinung ... so sekundär stattgefunden hat, wie die Funktion Spiel selbst primär genannt werden muß 
auch die Folge so sekundär, wie ... muß, stattgefunden bat, eintreten, also ein eingeschobener Satz. Daß diese Möglichkeit, die in unseren Belegen immerhin viermal gegeben war, nicht ein einziges Mal ausgenutzt wurde, spricht für die gegenwärtige Tendenz, möglichst "eins nach dem anderen zu erledigen" und deshalb die Nebensätze auszuklammern.

Als neunten Beleg haben wir einen Fall anzuführen, in dem man wegen der (mißglückten) syntaktischen Fügung kaum noch von einem Attributivsatz, sondern eher von einem isolierten Nebensatz sprechen kann:

6201 Genau wie die amerikanische Gesellschaft und der ... Volkscharakter ... sich tief von den europäischen unterscheiden, ebenso verschieden sind die politischen ... Entwicklungen ...

Hier ist das Vorfeld des Hauptsatzes durch ebenso verschieden vollständig besetzt, und der Vergleichssatz wird dadurch in die isolierte Spitzenstellung gedrängt. Allerdings hat dieser Beleg kaum Beweiskraft. Er ist aus dem Englischen übersetzt und scheint eine Konstruktion des Typs Exactly as ..., as different are ... nachzuahmen.

5. Durch einen einzigen Beleg ist der mit wie denn eingeleitete Vergleichssatz bezeugt:

6503 Hier ist uralte Uberlieferung ... am Werk, wie denn überhaupt die Tierriesen ein Reservat für uralte Überzeugungen vom Tierleben sind.

Dieser Fügungstyp scheint, nach der Seltenheit seines Vorkommens zu urteilen, modernem Sprachgebrauch kaum noch zu entsprechen. ${ }^{37}$ Der mit wie denn eingeleitete Nebensatz hat offenbar seinen Platz stets am Ende des Satzgefüges. Er läßt sich nicht als Satzglied in den übergeordneten Satz integrieren; dagegen könnte er als neuer Hauptsatz formuliert werden (... am Werk. Uberbaupt sind die Tierriesen ...). Wir haben es also nicht mit einem Glied- oder Teilsatz zu tun, sondern mit einem weiterführenden Nebensatz, und zwar einem weiterführenden Vergleichssatz.

6. Neben den bisher erörterten Typen des Vergleichssatzes gehört hierher eine sehr einheitlich geprägte und mit 36 Belegen stark vertretene Gruppe, die gleichwohl in den Grammatiken unbeachtet geblieben ist. Ich möchte sie als "vergleichenden Relativsatz" bezeichnen. Einige Beispiele: 
3337 An dem Wärmebegriff, wie er hier dargestellt wurde, bemerken wir ...

3143 Die Einheit der Wissenschaft gründet sich ... auf eine Gemeinschaft, wie ich sie beschrieben habe.

7202 Infolgedessen ist mit ... Mutationen durch die natürliche Radioaktivität zu rechnen, wie sie innerhalb und außerhalb der Organisation auftritt.

Völlig übereinstimmende gemeinsame Merkmale der drei aufgeführten und überhaupt aller hierher gehörigen 36 Sätze sind folgende:

a. Semantisch liegt ein (wenn auch abgeschwächter) Vergleich vor.

b. Überall läßt sich wie + Pronomen durch ein Relativpronomen ersetzen (Wärmebegriff, der dargestellt wurde - Gemeinscbaft, die ich beschrieben babe - Radioaktivität, die ... auftritt). Dabei tritt ähnlich wie bei der Transformation von modalen Aussagesätzen zu daß-Sätzen ${ }^{38}$ eine leichte semantische Veränderung ein.

c. Sie werden durch ein alleinstehendes wie eingeleitet. Pleonastisches so ist nur zweimal belegt (s. unten 4032).

d. Die wie-Sätze sind - wie die größte Gruppe der Relativsätze - auf ein Substantiv bezogen, dem sie in Kontaktstellung (vgl. 3337 und 3143) oder in Distanzstellung (7202) folgen. Es handelt sich demnach um Teil(Attribut-)Sätze.

e. Das Beziehungswort wird im wie-Satz pronominal durch eine Form des Pronomens er, sie, es oder (ziemlich selten) der Demonstrativpronomen der, die, das wieder aufgenommen, und zwar entweder als Subjekt (vgl. 3337, 7202) oder als Akkusativ-Objekt (3143).

Durch diese fünf Merkmale ist die Gruppe sehr deutlich gekennzeichnet und von anderen wie-Sätzen klar abzugrenzen.

Wie es bei den pronominalen Relativsätzen der Fall ist, sind auch diese vergleichenden relativen wie-Sätze in ihrer Stellung zum übergeorneten Satz beweglicher als alle vorher behandelten Gruppen. ${ }^{39} \mathrm{Als}$ Attributsätze, die einem Beziehungs-Substantiv folgen müssen, können sie zwar niemals allein das Vorfeld füllen. Aber im Anschluß an das Beziehungswort stehen sie in 7 Belegen im Vorfeld (vgl. das Beispiel 3337). Am Ende des Satzfeldes, darunter viermal an einen Nebensatz oder Infinitiv angeschlossen, finden sich 20 Belege, davon 11 in konti- 
nuierlicher (vgl. 3143), 9 in diskontinuierlicher Folge.

Einschub in das Satzfeld, stets kontinuierlich, wie in

4032 Dagegen sind Wasser und Luft, so wie sie ... im Boden enthalten sind, dessen ... Bestandteile ${ }^{40}$,

ist dreimal zu beobachten; Einschub in einen Nebensatz, wie in

6927 ... angesichts der Feststellung, daß unsere ... Parallelen mit ... Landschaften, wie die Maler sie uns vermittelt haben, nicht passen.

kommt sogar sechsmal vor. ${ }^{41}$ Es werden also alle Stellungen, die für Teilsätze überhaupt möglich sind, von den vergleichenden Relativsätzen in Anspruch genommen, wenn auch die Bevorzugung der Endstellung deutlich ist.

Als eines der Merkmale des vergleichenden Relativsatzes hatten wir seine Beziehung auf ein vorhergehendes Substantiv festgestellt. Davon weicht der folgende Beleg, der im übrigen Vergleichbares bietet, ab:

6088 Zerbrechlich und langgestreckt, wie sie war, hat man diese Verbindung einer ... Kette von Ameisen verglichen.

Der wie-Satz ist hier auf Adjektive bezogen, und sein Pronomen sie nimmt anaphorisch das Substantiv Verbindung vorweg, das man wohl im semantischen, aber nicht im syntaktischen Sinne als Beziehungswort bezeichnen kann. Dieser Beleg soll hier als eine Fügung sui generis verzeichnet werden, und er kann in seiner Vereinzelung kaum als im modernen Sprachgebrauch üblich gelten. ${ }^{42}$

7. Eine letzte Art von Vergleichssätzen muß aus semantischen Gründen (der Vergleichs-Charakter ist kaum noch spürbar) und syntaktisch wegen ihrer geringen Platzfestigkeit als eigene Gruppe klassifiziert werden. Wir meinen wie-Sätze vom Typ

1313 Charis ist, wie der Name sagt, das Freudige.

Nicht weniger als 72 Belege des Corpus gehören dieser Gruppe an. Sie sollen als "Hinweissätze" bezeichnet werden. ${ }^{43}$

Diese wie-Sätze lassen sich zu nominalen Gliedern transformieren (Charis ist nach Aussage des Namens das Freudige), sind also (mit ganz wenigen Ausnahmen) Gliedsätze, und zwar Adverbialsätze. 
Während die Vergleichsnebensätze im engeren Sinne vornehmlich den Platz am Ende des Satzfeldes oder - in zweiter Linie - im Vorfeld einnehmen und äußerst selten ins Satzfeld oder in einen Nebensatz eingeschoben werden, während auch die vergleichenden Relativsätze, wenn auch weniger strikt, diese Positionen bevorzugen, erweisen sich die Hinweissätze als nahezu stellungsfrei. So könnte Satz 1313 auch in den Formen Wie der Name sagt, ist Charis ... oder: ... ist das Freudige, wie der Name sagt auftreten. Solche Verschiebeproben könnten wir zwar auch mit anderen Klassen von wie-Sätzen anstellen. Entscheidend aber ist, daß dort solche Möglichkeiten im Sprachgebrauch nicht aktualisiert werden.

Die Hinweissätze bieten folgendes Bild: 11 Belege füllen das Vorfeld, ebenso viele stehen am Satzende. Dagegen wird die weit überwiegende Mehrzahl eingeschoben: 16 Belege bezeugen Einschub in einen Nebensatz, und nicht weniger als 34 Belege schieben den wie-Satz (wie oben 1313) in das Satzfeld des Hauptsatzes ein. Keine andere Art der im Corpus vertretenen Nebensätze weist einen so hohen Prozentsatz von Einschub-Stellungen auf. Gegenüber der ausgesprochenen Tendenz des heutigen Schriftdeutsch (die auch an anderen Textsorten als der populärwissenschaftlichen Prosa feststellbar ist), den Satzverlauf nicht durch Einschübe zu unterbrechen, bevorzugen die Hinweissätze diese Stellung vor allen anderen Möglichkeiten. Das liegt offenbar an ihrem geringen Aussagegewicht. Sie vermitteln beiläufige Informationen und können oft geradezu als parenthetische Einschübe betrachtet werden, so etwa in

3648 Die Konzeption eines allgemeinen ... Spielbegriffs hat, wie wir schon vorausschickten, erst spät stattgefunden.

Nicht selten werden sie auch durch Interpunktion als Parenthesen gekennzeichnet:

6016 Sie ... gegeneinander spielen zu lassen ist - wie wir oben gesehen haben - in der gegenständlichen Kunst typisches Mittel der Karikatur.

Es ist bekannt, daß an erster Stelle des Satzfeldes, gleich hinter dem Verbum finitum, das Beiläufige seinen Platz findet, und gerade dies ist die bevorzugte Stelle für die Hinweissätze: 23 haben hier ihren Platz, acht stehen an zweiter, drei an dritter Stelle im Satzfeld. 
In der Zahl von 72 Belegen sind drei inbegriffen, die sich der obigen Beschreibung nicht fügen. Sie richten sich nach folgendem Muster:

8907 Manche dieser Gefäße aus ... dünnwandigem Bucchero, wie man diese frühe Produktion nennt, zeigen ... Spuren einstiger Vergoldung.

Die semantische Funktion dieser Belege ist es, auf Fachausdrücke hinzuweisen. Sie haben dann ein "Beziehungswort", dem sie unmittelbar folgen müssen. Dieselbe Funktion hat auch das Partizip sogenannt (aus sogenannten dünnwandigem Bucchero). Da diese Transformation semantisch befriedigend und syntaktisch in jedem Fall möglich ist, halte ich für diese Subgruppe die Bezeichnung "attributive Hinweissätze" für angebracht.

\section{Ergebnisse}

Die sehr hohe Zahl der Hinweissätze (ein Drittel aller wie-Nebensätze) mag überraschen. Dafür ist nun gewiß die Textsorte, die das Material zu unserer Untersuchung lieferte, verantwortlich zu machen. Wissenschaftliche Prosa wird solcher Rück- und Vorverweise in viel höherem Maße bedürfen als andere Textsorten. In erzählender Prosa wird man diesen Satztyp gewiß sehr viel seltener finden. Ähnlich mag es beim vergleichenden Relativsatz und beim indirekten Fragesatz stehen. Umgekehrt sind etwa Modal- oder Vergleichsnebensätze in erzählender Prosa vermutlich häufiger anzutreffen als in (populär)wissenschaftlichen Texten. ${ }^{44}$

Das ganz vereinzelte Vorkommen gewisser Satztypen ließ uns auf den individuellen Sprachgebrauch aufmerksam werden. Darunter waren ein Goethe-Zitat und zwei Sätze von Autoren, die noch im 19. Jahrhundert geboren wurden. Jeder Leser nimmt solche Fügungen mit vollem Verständnis auf; es bleibt aber fraglich, ob sie von jüngeren Autoren heute noch aktualisiert werden, ob sie also noch dem heutigen Sprachstil entsprechen. Systematische Beobachtungen solcher Art könnten auf sonst kaum bemerkbare Wandlungen im syntaktischen System aufmerksam machen und zur Präzisierung von Pauschalurteilen wie "veraltet" oder "ungebräuchlich" führen. 
Eine systematisch wichtige Erkenntnis, die allerdings weiterer Erforschung bedarf, wurde in der Aufdeckung von "isolierten Nebensätzen" 45 gewonnen. Sie stellen neben Glied-, Teil- und weiterführenden Sätzen eine vierte Kategorie von Nebensätzen dar und beweisen erneut, daß der Terminus "Gliedsatz", wenn er definierende Bezeichnungskraft haben soll, nur auf eine Untergruppe von Nebensätzen angewendet werden darf. Im übrigen wäre zugleich mit den isolierten Nebensätzen wohl auch dem Problem der parenthetischen Sätze näher zu kommen. Unsere Untersuchung konnte, indem sie ins Einzelne ging, auf semantische und syntaktische Phänomene aufmerksam machen, die in den großen zusammenfassenden Grammatiken zum Teil gar nicht oder doch nur oberflächlich dargestellt werden. Das ist ihren Verfassern nicht zum Vorwurf zu machen. Eine Gesamtdarstellung kann immer nur die großen Linien nachziehen. Außerdem fehlt es bei sehr vielen Problemen (nicht nur bei dem in dieser Studie behandelten) an den vorbereitenden Einzeluntersuchungen, die erst die Grundlage zur umfassenden Darstellung liefern können. Möge sich in dieser Hinsicht diese Studie als nützlich erweisen, auch indem sie über Thesen, die hier aufgestellt, und neue Termini, die eingeführt werden, die Diskussion anregt.

A n m erkungen

1 Das Corpus umfaßt aus je 1000 Sätzen von 50 Autoren populärwissenschaftlicher Prosa (Rowohlts Deutsche Enzyklopädie) sämtliche Sätze bestimmter Länge, gezählt nach Wörtern. Zusammensetzung, Umfang und Einrichtung sind wiederholt beschrieben worden, vgl. z.B. Studium Generale 15 (1962), S. 49 - 59. Eine abermalige Beschreibung erscheint unnötig.

2 Anstelle vieler Grammatiken sei hier nur exemplarisch verwiesen auf $P$ aul Grebe u.a., Duden-Grammatik der deutschen Gegenwartssprache, 2. Aufl., Mannheim 1966 und Johannes Erben, Abriß der deutschen Grammatik, von mir benutzt in 7. Aufl., Berlin 1964. In beiden Werken sind im Wortregister unter wie die einschlägigen Stellen nachgewiesen.

3 Dieser Satz ist im heutigen Deutsch jedenfalls möglich. Allerdings ist in schriftsprachlicher Formulierung eher zu erwarten: Wie sebr bat er sich gefreut. 
5 Alle Belege aus dem Corpus werden mit ihrer Katalognummer aufgeführt. Im Corpus sind weiterhin Band- und Seitenzahl angegeben, so daß der Autor ermittelt und die Distribution kontrolliert werden kann.

Auf die Unterscheidung zwischen Partizipialadjektiv (zartbesaitet), das wir unter die Klasse der Adjektiva subsumieren, und dem echt verbalen Partizip einzugehen, ist hier kein Anlaß.

7 Endstellung des Verbum finitum wäre in den drei folgenden Belegen syntaktisch möglich.

8 Daß Konjunktionen wie und, oder, denn dem Vorfeld vorgeschaltet werden können und im syntaktischen System die Erststellung des vorfeldfuillenden Satzgliedes nicht beeinträchtigen, ist opinio communis aller Grammatiker.

9 Die Umwandlung in einen Fragehauptsatz (Wie schön sind die Gebärden ...?) gibt, sofern sie überhaupt möglich ist, den fünf Sätzen eine andere Bedeutungsrichtung.

10 S. u. S. $166 \mathrm{ff}$,

11 Möglich ist natürlich auch die Bindung an ein Adjektiv (Typen: Wie alt ist er?); aber sie ist in unserem Corpus nicht belegt.

12 Eine semantische Untergliederung der Gruppe scheint möglich. Statt auf welcbe Weise, das in allen zehn Belegen eingesetzt werden könnte, ist sechsmal auch der Ersatz durch aus welcher Ursache, also eine Kausalfrage möglich, in den vier anderen Fällen könnte man fragen: Mit welcben Mitteln (vgl. 4537 Wie kann man etwas über dieses Ereignis erfabren?). Hier liegt also eine Instrumentalfrage vor. Eine genauere Untersuchung muß hier unterbleiben, da nicht genügend Material zur Verfügung steht.

13 Zu der größeren Gruppe ist auch folgender Satz gezählt: 6712 Icb möchte ... bei der Frage verweilen: Wie konnte das ... Publikum eine solcb niedrige Vorstellung ... sich gefallen lassen? Der äußeren Form nach handelt es sich um einen Fragehauptsatz, der übrigens auch als Ausrufsatz aufgefaßt werden könnte; nach dem syntaktischen Bezug stellt er dagegen ein Attribut (oder eine Apposition) zu Frage dar, müßte also insofern unter die Nebensätze eingereiht werden.

14 Wohl aber natưrlich gradhöhere Nebensätze, so daß weitere Nebensätze zweiten, dritten Unterordnungsgrades von ihnen abhängen können. 
Im Zusammenhang eines Kontextes kann im semantischen Sinne auch ein Hauptsatz als "Vergleichssatz" angesehen werden. Ein Satz wie So bat es sein Vater auch getrieben hat Vergleichscharakter und ist nur aus dem übersatzmäßigen Kontext voll zu verstehen.

16 Vgl. zu dieser Transformation oben Anm. 3.

17 S. unten S. 167.

18 Ich fühle mich versucht, dieses wie der Klasse der Konjunktionen zuzuordnen, lasse diese Frage aber dahingestellt. Immerhin könnte man in dem oben zitierten Satz 1908 das wie durch auf welche Weise ersetzen, also durch eine adverbiale Bestimmung, und das ist bei anerkannten Konjunktionen wie daß, als, weil niemals der Fall. Trotzdem scheint mir wie in abhängigen Fragesätzen kein Adverb zu sein. Man wird die nebensatzeinleitenden Konjunktionen letzten Endes in mehrere Subklassen zu unterteilen haben.

19 Daß modifizierendes wie beim Adjektiv usw. in unserem Corpus nicht im Vorfeld belegt ist, mag Zufall sein. Der oben zitierte Satz 1496 wäre auch vorfeldfüllend durch Wie weit das stimmte, ist . . scbwer festzustellen realisierbar.

20 Es muß bemerkt werden, daß 11 dieser abhängigen Fragesätze einem Infinitiv (vgl. den oben zitierten Satz 3529) und nur zwei einem konjunktionalen Nebensatz untergeordnet sind.

21 Die Prinzipienfrage, ob Elemente des Hauptsatzes wie oben darüber, dar $u m$ als die eigentlichen Satzglieder und die wie-Sätze als Attribute dazu, also als Gliedteilsätze aufzufassen sind, oder ob - wie im Text angenommen - die wie-Sätze den Kern des Gliedes ausmachen, kann hier nicht entschieden werden. Daß das Adverb gelegentlich fehlen kann (4234 Es bat keinen Sinn, weiter zu fragen, wie die Anziebung ... zustande kommt. nacb dem Zustandekommen zu fragen. - Möglich: danach zu fragen, wie...), spricht für die im Text geäußerte Auffassung.

22 Ich würde es in diesem Fall und in den beiden anderen Belegen (7198 Hypothesen ..., wie ... ein derartiger Vorgang sich abspielen könnte und 8534 Möglicbkeiten, wie das Ammoniak mit organiscben Säuren verbunden ... werden kann) nicht für richtig halten, sie als Relativsätze zu klassifizieren.

23 In 4439 und in 7198 ( $\mathrm{vgl}$. Anm. 22) steht der Attributivsatz in Distanzstellung zum Beziehungswort außerhalb des Satzrahmens. Die Endstellung ist hier umso bemerkenswerter, als Einbeziehung in das Satzfeld möglich wäre: scbließt die Frage, wie sie in Erscheinung treten, ein. Im heutigen Schriftdeutsch genießt die Endstellung bei weitem den Vorzug. 
Endstellung wird auch in den drei Fällen angenommen, in denen auf den wie-Satz noch ein von ihm abhängiger Nebensatz 2. Grades folgt, z.B. 3824: Die Hartnackigkeit ... beweist, wie wichtig es ibnen war, daß dieses Beispiel keine Nachabmung fand.

25 Vgl. auch das oben S. 164 ausgeführte Beispiel wie unrealistiscb / daß ... unrealistisch

26 Hier liegt wieder ein indirekter Fragesatz vor.

27 S. unten S. 172.

28 In 6159 und 8299 betont auch noch die Zeichensetzung (Gedankenstrich statt Komma) die Sonderstellung des Einschränkungssatzes.

29 Studien zur Syntax des heutigen Deutsch (= Sprache der Gegenwart, Band 6), Düsseldorf 1970, S. 85 f.; zu den weiterführenden Nebensätzen vgl. auch Duden-Grammatik $\$ \$ 6385$ - 6400 .

30 Als solche könnten sie auch nicht bezeichnet werden, wenn sie am Ende des Satzgefüges ständen.

31 Diese Bezeichnung ist vorbereitet, aber nicht verwendet durch Schulz-Griesbach, Grammatik der deutschen Sprache (von mir benutzt in der 1. Aufl., München 1960), S. 292, § G 584. Dort wird auf die durch $a b$... oder eingeleiteten Konsekutivsätze hingewiesen, die zwar als Gliedsätze bezeichnet werden, zu denen aber auf S. 278, $\$$ G 388 , mit Recht festgestelit wird: "Dieser konzessive Gliedsatz ist nicht Satzglied des folgenden Hauptsatzes". Die Fortsetzung allerdings, "sondern ein Gliedsatz im Nachfeld eines gedachten Satzes" des Typs es ist gleichgültig, ob ... (bzw. wie) halte ich für eine ad-hoc-Erklärung, die dem syntaktischen System nicht gerecht wird.

32 Vgl. z.B. 1006 Blaue Dreiecke sind wie nächtliche Berge oder Hausdächer.

33 So ließe sich der Beleg 6300 transformieren zu: Nach Art eines sich die Rosinen ... beraussuchenden Feinscbmeckers hat die Zecke ..., oder auch Nach Art eines Feinscbmeckers, der ...

34 Dabei ist zu bedenken, daß es sich bei dem wie-Satz um ein "entbehrliches" Element handelt. Der Satz 3934 wäre auch ohne ihn syntaktisch vollständig.

35 Die allerdings neu zu untersuchen und zu präzisieren wäre. Dabei wäre auch die diskontinuierliche Möglichkeit in Betracht zu ziehen: So ungewóbnlich ist es nicht, wie es scheinen mag. 
36 So könnte in allen sechs Belegen völlig fehlen. Auch wäre die Fügung könnte so variieren, wie ... denkbar. Aber diese diskontinuierliche Stellung ist überhaupt nur ein einziges Mal belegt: $\mathbf{9 4 3 1}$... sich jedem Ding gegenüber so zu verbalten, wie sein Wesen es fordert.

37 Der Autor des Satzes ist 1897 geboren.

38 S. oben S. 164.

39 Auf die Verhältnisse in den pronominalen Relativsätzen einzugehen, ist hier nicht der Ort, Daß der Vergleich zulässig ist, lehren mich meine bisherigen Beobachtungen.

404032 ist einer der beiden Belege mit so wie.

41 Generell ist festzustellen: Eingeschobene Nebensätze sind im modernen Schriftdeutsch ziemlich selten. (Teilsätze werden eher eingeschoben als Gliedsätze). Dabei ist der Einschub in Nebensätze (wie in 6927) relativ häufiger als der in Hauptsätze (wie in 4032).

42 Der Autor - der einen sehr eigenwilligen Stil schreibt - ist 1898 geboren. (vgl. Anm. 37).

43 In der Gesamtzahl von 72 sind übrigens sechs verkürzte Hinweissätze des Typs wie gesagt, wie festgestellt enthalten.

44 Wir sind hier auf Vermutungen angewiesen; Zählungen liegen nicht vor.

45 S. oben S. 170 und 173. 